\title{
TU/e EN⿴HONE

\section{Consequences of plasma rotation for neoclassical tearing mode suppression by electron cyclotron current drive}

Citation for published version (APA):

Ayten, B., \& Westerhof, E. (2012). Consequences of plasma rotation for neoclassical tearing mode suppression by electron cyclotron current drive. Physics of Plasmas, 19(9), 092506-1/11. [092506].

https://doi.org/10.1063/1.4751873

DOI:

10.1063/1.4751873

Document status and date:

Published: 01/01/2012

\section{Document Version:}

Publisher's PDF, also known as Version of Record (includes final page, issue and volume numbers)

\section{Please check the document version of this publication:}

- A submitted manuscript is the version of the article upon submission and before peer-review. There can be important differences between the submitted version and the official published version of record. People interested in the research are advised to contact the author for the final version of the publication, or visit the $\mathrm{DOI}$ to the publisher's website.

- The final author version and the galley proof are versions of the publication after peer review.

- The final published version features the final layout of the paper including the volume, issue and page numbers.

Link to publication

\section{General rights}

Copyright and moral rights for the publications made accessible in the public portal are retained by the authors and/or other copyright owners and it is a condition of accessing publications that users recognise and abide by the legal requirements associated with these rights.

- Users may download and print one copy of any publication from the public portal for the purpose of private study or research.

- You may not further distribute the material or use it for any profit-making activity or commercial gain

- You may freely distribute the URL identifying the publication in the public portal.

If the publication is distributed under the terms of Article 25fa of the Dutch Copyright Act, indicated by the "Taverne" license above, please follow below link for the End User Agreement:

www.tue.nl/taverne

Take down policy

If you believe that this document breaches copyright please contact us at:

openaccess@tue.nl

providing details and we will investigate your claim. 


\title{
Consequences of plasma rotation for neoclassical tearing mode suppression by electron cyclotron current drive
}

\author{
B. Ayten ${ }^{\text {a) }}$ and E. Westerhof \\ FOM Institute DIFFER — Dutch Institute for Fundamental Energy Research, Association EURATOM-FOM, \\ Trilateral Euregio Cluster, PO Box 1207, 3430 BE Nieuwegein, The Netherlands
}

(Received 25 June 2012; accepted 23 August 2012; published online 13 September 2012)

In the generalized Rutherford equation describing the nonlinear evolution of the width of the magnetic island associated with a neoclassical tearing mode, the effect of localized current drive is represented by a term $\Delta_{C D}^{\prime}$. We investigate oscillations in $\Delta_{C D}^{\prime}$ originating from the rotation of the island through the electron cyclotron power deposition region and their dependence on the collisional time scale on which the driven current is generated, the rotation period, the island size, and the power deposition width. Furthermore, their consequences for the island growth or the stabilization are analyzed. This work shows that the net result of the oscillations in $\Delta_{C D}^{\prime}$ is a slight increase in the stabilizing effect of electron cyclotron current drive and consequently, a reduction in the minimum power requirement to fully suppress an island.

[http://dx.doi.org/10.1063/1.4751873]

\section{INTRODUCTION}

Economic fusion reactors require operation at high $\beta$, the ratio of kinetic over magnetic pressure. However, high $\beta$ plasmas are prone to a number of magnetohydrodynamic (MHD) instabilities. Neoclassical tearing modes (NTMs) produce magnetic islands around resonant surfaces with rational safety factor $q=m / n$, where $m$ is the poloidal and $n$ is the toroidal mode number. They lead to confinement degradation and even to disruptions. Thus, control of NTM amplitudes is important in order to optimize the efficiency and the performance of a tokamak fusion reactor.

NTMs are sustained by the annihilation of the pressuregradient driven bootstrap current inside the magnetic island due to the pressure flattening across the island. ${ }^{1,2}$ Suppression of the NTM can be achieved through the replacement of this missing bootstrap current either non-inductively by localized current drive or inductively by localized heating reducing the resistivity and thus increasing the inductively driven current density. Because of the good localised power and driven current profiles of electron cyclotron resonance heating (ECRH) and electron cyclotron current drive (ECCD), they are the preferred tools for the stabilization of NTMs. ${ }^{3}$ NTM suppression experiments have been performed successfully using both ECRH and ECCD. ${ }^{4-14}$

The generalized Rutherford equation (GRE) $)^{15,16}$ provides a theoretical model for the growth and stabilization of tearing modes. It describes the nonlinear temporal evolution of the full width $w$ of the associated magnetic island as a function of different stabilizing and destabilizing mechanisms. ${ }^{2,16-20}$ Including only the most relevant terms in the context of the present paper, the GRE is written as

$$
0.82 \frac{\tau_{r}}{r_{s}} \frac{d w}{d t}=r_{s} \Delta_{0}^{\prime}(w)+r_{s} \Delta_{b s}^{\prime}+r_{s} \Delta_{C D}^{\prime},
$$

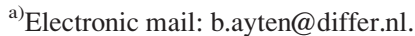

where $\tau_{r}=\mu_{0} r_{s}^{2} / \eta$ is the current diffusion time for the plasma resistivity $\eta$ at the resonant surface $r_{s}$ of the island. $\Delta_{0}^{\prime}(w)$ is the classical stability index. The driving mechanism of NTMs, which is the perturbation of the bootstrap current, is represented by the second term. The last term refers to the stabilizing effect of localized current drive. In this work, we neglect the effect of heating associated with ECCD and a possible modification in equilibrium current profile as a consequence of the non-inductive current drive which can modify $\Delta_{0}^{\prime}$. Moreover, we exclude finite island size effects on the classical stability index ${ }^{21}$ and the stabilizing Glasser-Green-Johnson effect resulting from the magnetic field curvature. ${ }^{22}$

Conventionally, the $\Delta_{C D}^{\prime}$ term is obtained by averaging the effect of the ECCD over an island rotation period $\tau_{\text {rot }}$. The island rotation period $\tau_{\text {rot }}$ as used here is defined as the period of rotation of the magnetic island as observed by a fixed external observer or structure such as an ECCD antenna. Formally, this requires the assumption that the rotation period is much shorter than the collisional time scale $\tau_{\text {coll }}$ on which the electron cyclotron (EC) driven current is generated/decays. When this assumption is not valid and the rotation period is of the same order or longer than the relevant collisional time scale, the EC driven current $\delta J_{C D}$ profile becomes time dependent, oscillating and moving through the island with the rotation period. Current centered at the O-point of a rotating island stabilizes the island more efficiently, whereas $\mathrm{CD}$ on the island X-point has a destabilizing effect. As a result, $\Delta_{C D}^{\prime}$ will oscillate with the rotation period as well.

Another time scale related to the stabilization of islands is $\tau_{N T M}$, the characteristic time scale for island evolution. When the island evolution is slow, the time average of $\Delta_{C D}^{\prime}$ again results in the conventional expression for $\Delta_{C D}^{\prime}$. However, when the rotation is slow and the island evolves significantly during a rotation period, this is no 
longer true, and the oscillations of $\Delta_{C D}^{\prime}$ must be accounted for in the temporal evolution of the island. So far, little attention has been paid to the time dependency of the $\Delta_{C D}^{\prime}$ term and its consequences for the island evolution. It would thus be of interest to analyze the $\Delta_{C D}^{\prime}$ oscillations and their dependence on the different time scales, the island size and power deposition width. Furthermore, it is of interest to study the consequences of the $\Delta_{C D}^{\prime}$ oscillations for the island evolution and in particular for the minimum power requirement to fully suppress an island. This might especially be relevant in case of relatively slow rotation as is the case for the next generation fusion devices, like ITER. In the present-day, tokamaks like ASDEX Upgrade, JT-60U, and DIII-D, the rotation is much faster owing to neutral beam injection (NBI). However, present day experiments could access the low rotation regime by balanced beam injection or coupling to slowly rotating resonant magnetic perturbations.

This paper studies how the oscillation in $\Delta_{C D}^{\prime}$ is determined by the two time scales, $\tau_{\text {rot }}$ and $\tau_{\text {coll }}$ and additionally, how it affects the growth or stabilization of the island on its evolutionary time scale $\tau_{N T M}$. This is analyzed in the parameter space spanned by the three time scales. When $\tau_{\text {coll }}$ and $\tau_{N T M}$ are normalized to $\tau_{\text {rot }}$, a two dimensional normalized parameter space results. In this parameter space, we recognize two limits. On one hand, the regime of very fast rotation $\left(\tau_{N T M} / \tau_{\text {rot }} \gg 100\right)$ in which $\Delta_{C D}^{\prime}$ is obtained through an average over a rotation period. This so-called standard regime has been studied extensively. ${ }^{17,23-25}$ On the other hand, one has the locked mode regime $\left(\tau_{N T M} / \tau_{\text {rot }} \ll 1\right)$ in which the island does not rotate significantly during its evolution. This work focuses on the parameter space between these two extrema. In addition, relevant results for the locked mode regime are included. It should be noted that in this parametric study, we will vary $\tau_{\text {coll }}$ and $\tau_{N T M}$ independent of each other. In a practical experiment, these two time scales are of course strongly linked.

Dynamic effects from the finite collision time and island rotation have been studied before by Giruzzi et al. ${ }^{26}$ in the context of lower hybrid current drive (LHCD) and ECCD stabilization of NTMs in ITER. The present paper supplements this work by providing a systematic analysis over the full range of the relevant parameter space and by including a self consistent modelling of the driven current evolution inside an evolving and rotating magnetic island.

The plan of this paper is as follows. Section II presents and discusses the GRE taking into account all the relevant terms to be used in the present work. The term expressing the effect of the current drive in the GRE is used as given in Refs. 17, 24, and 25. An equation describing the time evolution of $J_{C D}$ in the presence of an evolving and rotating island is presented. The time-dependent current density introduced by the island rotation is incorporated into the generic $\Delta_{C D}^{\prime}$ term. In Sec. III, the resulting time dependent ECCD term is presented for the case of constant island width. This is followed in Sec. IV by a study of its effect on the island evolution. The relevance of these results for present day and future tokamak experiments is discussed. Finally, Sec. V summarizes the conclusions.

\section{THE MODEL}

\section{A. Generalized Rutherford equation}

The modelling of the island width evolution discussed in this paper is based on the GRE as written in Eq. (1) taking into account only the most relevant terms in the context of the present work. Both the second and third term on the right hand side arise from a perturbation of the non-inductive current density. The modification to parallel Ohm's law as a result of such a perturbation to the non-inductive current density, $\delta j_{n i}$, results in a contribution to the GRE of the generic form

$$
r_{s} \Delta_{n i}^{\prime}=-\frac{16 \mu_{0} L_{q} r_{s}}{B_{p} \pi w^{2}}\left[\int_{-\infty}^{\infty} d x \oint d \xi \delta j_{n i} \cos \xi\right],
$$

where $L_{q}=q / q$ is the shear length and $B_{p}$ the poloidal magnetic field at the rational surface. The second term on the right hand side of Eq. (1) describes the driving term from the helically perturbed bootstrap current. As the bootstrap current is annihilated by the fast parallel transport inside the island, the corresponding perturbation to the non-inductive current is $\delta j_{n i}=-J_{B S}$ everywhere inside the island, where $J_{B S}$ is the bootstrap current density at the rational surface of the mode in the unperturbed equilibrium. The resulting bootstrap term is then written as ${ }^{2,24,27,28}$

$$
r_{s} \Delta_{b s}^{\prime}=\frac{16 \mu_{0} L_{q} r_{s}}{B_{p} \pi} \frac{4 J_{B S}}{3 w} \frac{w^{2}}{w^{2}+w_{\text {marg }}^{2}},
$$

where the factor $4 / 3$ follows from the evaluation of the integrals inside the island only and the factor $w^{2} /\left(w^{2}+w_{\text {marg }}^{2}\right)$ is introduced to account for the effects of incomplete flattening of the pressure inside small islands, as a consequence of the competition between parallel and perpendicular transport in the island region. ${ }^{27}$ As a consequence, the bootstrap current is only partially destroyed in the case of small islands, which limits the instability drive in the small island limit. The parameter $w_{\text {marg }}$ is defined as the island width at which the bootstrap term has its maximum. Other forms of this factor were also proposed representing different stabilizing contributions in the small island limit. ${ }^{29}$ For the present work, these different models would not change the results qualitatively.

The classical stability index $\Delta_{0}^{\prime}$ can be calculated in an indirect way by relating it to the island size, $w_{s a t}$ at which the NTM growth saturates in the absence of other stabilizing effects. Since at $w_{\text {sat }}$, the growth rate of the island without ECCD is zero,

$$
\Delta_{0}^{\prime}=-\Delta_{b s}^{\prime}\left(w=w_{s a t}\right) .
$$

Possible additional dependencies of $J_{B S}$ and $\Delta_{0}^{\prime}$ on the island width $w$ are neglected. Also these would not have a qualitative effect on the results presented in the present paper.

The stabilizing contribution of the EC driven current density $\delta j_{n i}=J_{C D}$, in the GRE is ${ }^{17,24,25}$

$$
r_{s} \Delta_{C D}^{\prime}=-\frac{16 \mu_{0} L_{q} r_{s}}{B_{p} \pi w^{2}}\left[\int_{-\infty}^{\infty} d x \oint d \xi J_{C D} \cos \xi\right]
$$


where $x \equiv r-r_{s}$ is the radial coordinate relative to the rational surface and $\xi$ is the helical angle in the cross-section of the island. To evaluate this term as a function of time, a proper equation for the evolution of the driven current density is needed taking into account the effects of rotation and a finite collision time as well as the effect of the evolution of the island. Once $J_{C D}$ is known, $\Delta_{C D}^{\prime}$ is obtained through direct numerical evaluation of the integrals without further approximations.

As the purpose of this work is to obtain a generic understanding of possible effects of the $\Delta_{C D}^{\prime}$ oscillations on the island evolution and of their dependence on the different time scales involved, it is useful to write a normalized GRE. To this end, we identify the characteristic time scale for island evolution with the inverse growth rate coming from the maximum of the bootstrap term, i.e.,

$$
\tau_{N T M} \equiv \frac{0.82 \tau_{r}}{r_{s} \Delta_{b s, \max }^{\prime}}
$$

Island sizes and deposition widths are then normalized by $r_{s}$ and time by $\tau_{N T M}$. This way the GRE is obtained in its following normalized form:

$$
\frac{d \bar{w}}{d \bar{t}}=-\frac{2 \bar{w}_{\text {sat }} \bar{w}_{\text {marg }}}{\bar{w}_{\text {sat }}^{2}+\bar{w}_{\text {marg }}^{2}}+\frac{2 \bar{w} \bar{w}_{\text {marg }}}{\bar{w}^{2}+\bar{w}_{\text {marg }}^{2}}+\bar{\Delta}_{C D}^{\prime},
$$

where $\bar{t} \equiv t / \tau_{N T M}, \bar{w} \equiv w / r_{s}$, and $\bar{\Delta}_{C D}^{\prime}=\Delta_{C D}^{\prime} / \Delta_{b s, \max }^{\prime}$.

\section{B. The evolution of $J_{C D}$}

The dynamical modelling of the electron cyclotron current drive is performed as follows. A normalized Gaussian distribution is assumed for the radial power deposition profile

$$
P_{E C}=P_{t o t} \tilde{p}_{C W}(x, \xi) \mathcal{M}(t, \mathcal{D})
$$

with

$$
\tilde{p}_{C W}(x, \xi)=\frac{\Pi(\xi)}{\Delta \xi \pi^{3 / 2} w_{d e p} R r_{s}} e^{-4\left(x-x_{d e p}\right)^{2} / w_{d e p}^{2}}
$$

where $P_{\text {tot }}$ denotes the total injected power in case of continuous wave $(\mathrm{CW})$ application, $\xi$ the helical angle, $\Delta \xi$ the helical power deposition width, $R$ the tokamak major radius, $w_{\text {dep }}$ the full $e^{-1}$ power density width, and $x_{d e p}=r_{d e p}-r_{s}$ the deposition location relative to the resonant radius. As the island rotates, the power is deposited at different phases of the island with a helical width $\Delta \xi$. Under the assumption that at $t=0$, the helical profile is centered at $\xi=0$, this effect is included by the top hat function $\Pi(\xi)$ as

$\Pi(\xi)=1, \omega_{\text {rot }} t-\Delta \xi / 2 \leq \xi \leq \omega_{\text {rot }} t+\Delta \xi / 2$, modulus $2 \pi$,

$$
\Pi(\xi)=0, \text { otherwise, }
$$

where $\omega_{\text {rot }}=2 \pi / \tau_{\text {rot }}$ is the angular rotation frequency of the island. $\mathcal{M}(t, \mathcal{D})$ represents the power modulation and it is written in terms of a Heaviside function as

$$
\mathcal{M}(t, \mathcal{D})=H\left(\cos \left(\omega_{\text {rot }} t\right)-\cos (\mathcal{D} \pi)\right)
$$

where $\mathcal{D}$ is the power on-time fraction. It is assumed here that the power is modulated exactly at the island rotation frequency and that the power-on phase is exactly centered around the O-point passage through the ECCD beam.

At this point, we need to define the symmetric island topology adopted for this work: $\Omega=8 x^{2} / w^{2}-\cos \xi$ is the normalized flux surface label with $\Omega=-1$ at the O-point of the island and $\Omega=1$ at the X-point. Here, $x=r-r_{s}$ is the displacement from the resonant surface. Outside the island, the label $\sigma=\operatorname{sgn}(x)$ distinguishes the two different surfaces with identical $\Omega$ on opposite sides of $r_{s}$. Using the same notation as in Refs. 17, 25, and 27, the flux surface average of a function A can be defined as $\langle A\rangle \equiv\{A\} /\{1\}$, where

$$
\{A(\sigma, \Omega, \xi)\} \equiv \oint \frac{\mathrm{d} \xi}{2 \pi} \frac{w}{4 \sqrt{2}} \frac{A(\sigma, \Omega, \xi)}{\sqrt{\Omega+\cos \xi}},
$$

for $\Omega>1$, and

$$
\{A(\sigma, \Omega, \xi)\} \equiv \int_{-\hat{\xi}}^{\hat{\xi}} \frac{\mathrm{d} \xi}{2 \pi} \frac{w}{4 \sqrt{2}} \frac{[A(\sigma, \Omega, \xi)+A(-\sigma, \Omega, \xi)]}{\sqrt{\Omega+\cos \xi}},
$$

for $\Omega \leq 1$.

The parallel transport is assumed to be virtually instantaneous, such that the driven current density can be taken as a flux function. In that limit, the evolution of the driven current density is given by the bounce-averaged, quasi-linear Fokker-Planck equation. This equation describes the evolution of the bounce-averaged electron distribution function under the influence of both collisions and the EC driven quasi-linear diffusion. ${ }^{30-32}$ Because the island evolution is incompressible, the evolution of the island will not affect the bounce-averaged distribution function $f_{e}\left(S_{\Omega}\right)$ as a function of the total area enclosed by the flux surface as indicated in Fig. 1. Only on those surfaces that are being reconnected due to island growth or get disconnected due to island shrinkage, the distribution function must either be averaged over the two contributing surfaces or distributed equally over the two resulting surfaces. An equation for the evolution of the current density can be obtained by taking the first moment of the bounce-averaged, quasi-linear Fokker-Planck equation. In our model, we approximate the resulting equation as

$$
\begin{aligned}
\frac{\partial J_{C D}\left(S_{\Omega}, \sigma, t\right)}{\partial t}= & -\frac{J_{C D}\left(S_{\Omega}, \sigma, t\right)}{\tau_{\text {coll }}} \\
& +2 \pi R \frac{\eta_{C D}}{\tau_{\text {coll }}}\left\langle P_{E C}\right\rangle\left(S_{\Omega}, \sigma, t\right),
\end{aligned}
$$

where the first term on the right hand side describes the effects of collisions and the second term represents the current drive as a consequence of the quasi-linear EC diffusion. The collision time represents the current destruction rate ${ }^{33}$ and can be obtained from bounce-averaged, quasi-linear Fokker-Planck simulations. It will be strongly dependent on the velocity of the resonant electrons carrying the EC driven current. In the second term, the current drive efficiency is 


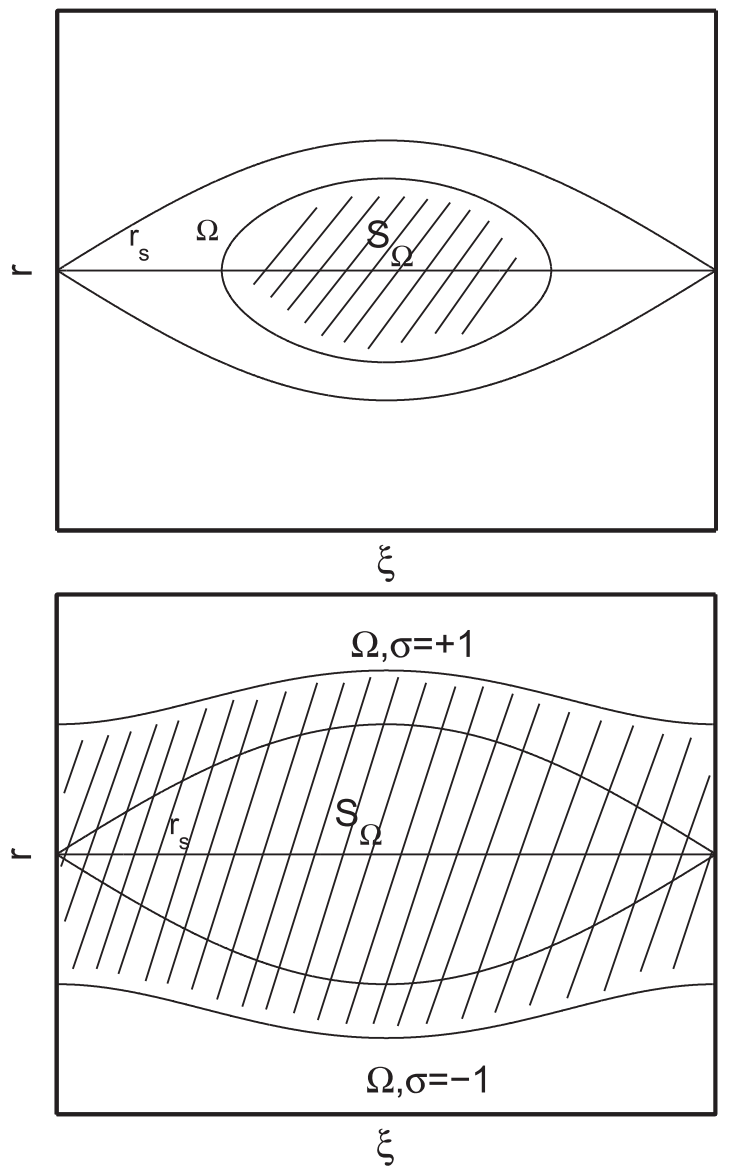

FIG. 1. $S_{\Omega}$ is the surface area enclosed by $\Omega . \sigma=+1$ represents $\Omega$ at a radial location $r>r_{s}$, whereas $\sigma=-1$ represents $\Omega$ at $r<r_{s}$.

assumed to be a simple constant, $\eta_{C D} \equiv I_{C D} / P_{t o t}$, where $I_{C D}$ is the total driven current obtained from either bounceaveraged, quasi-linear Fokker-Planck simulations or ray tracing calculations including adjoint calculation of the driven current. This neglects any detailed spatial dependence of the current drive efficiency over the region of power deposition. $\left\langle P_{E C}\right\rangle$ is the flux surface averaged power density. Also possible nonlinearities in $\eta_{C D}$ at high power densities are neglected.

In the limit of $\tau_{\text {coll }}=0$, the solution for the current density Eq. (15) becomes $J_{C D}\left(S_{\Omega}, \sigma, t\right)=2 \pi R \eta_{C D} P_{E C}\left(S_{\Omega}, \sigma, t\right)$ which corresponds to the assumption of instantaneous generation of the driven current. Equation (15) must be supplemented by a set of equations describing the effect of the island evolution: i.e., the effect of reconnection (for a growing island) or disconnection (for a shrinking island). On reconnected flux surfaces as a consequence of island growth,

for $S_{\text {sep }}\left(t^{-}\right)<S_{\Omega} \leq S_{\text {sep }}\left(t^{+}\right)$,

$$
\begin{aligned}
J_{C D}\left(S_{\Omega}, t^{+}\right)= & \frac{1}{2}\left(J_{C D}\left(S_{\Omega}, \sigma=1, t^{-}\right)\right) \\
& +\frac{1}{2}\left(J_{C D}\left(S_{\Omega}, \sigma=-1, t^{-}\right)\right),
\end{aligned}
$$

where $S_{\text {sep }}$ represents the enclosed surface area within the separatrix and $t^{-}$and $t^{+}$refer to the time steps in the simulation just before and just after the surfaces are reconnected, respectively, i.e., the current density is the average of the two current densities on the surfaces that have formed the newly reconnected surface. On flux surfaces that get disconnected as a result of island shrinkage,

$$
\begin{aligned}
& \text { for } S_{\text {sep }}\left(t^{+}\right)<S_{\Omega} \leq S_{\text {sep }}\left(t^{-}\right), \\
& \qquad J_{C D}\left(S_{\Omega}, \sigma= \pm 1, t^{+}\right)=J_{C D}\left(S_{\Omega}, t^{-}\right),
\end{aligned}
$$

where $t^{-}$and $t^{+}$now refer to the time steps just before and just after the surfaces are disconnected, i.e., the current density on surfaces that get disconnected is conserved and distributed equally over the two daughter surfaces on opposite sides of $r_{s}$. Equations (15)-(17) describe the model we use for the time dependent evolution of the driven current in the case of an evolving island. This set of equations is solved by means of a numerical code implemented in MATLAB, and solved concurrently with the island evolution according to the GRE.

\section{RESULTS OF $\Delta_{C D}^{\prime}$ CALCULATIONS: CONSTANT ISLAND WIDTH}

In this section, we solve Eqs. (5) and (15) for constant island width $w$ and power on-time fraction $\mathcal{D}=100 \%$. We look into the effects of the choice of helical width and $\tau_{\text {coll }} / \tau_{\text {rot }}$ on the time dependence of $\Delta_{C D}^{\prime}$. Moreover, these calculations are performed for a number of island widths to see the dependence of the results on the island and deposition widths. The conclusions drawn in this section will help to understand the results of the dynamical modelling with self consistent island width evolution given in Sec. IV. The results of the $\Delta_{C D}^{\prime}$ calculations will be normalized to a reference case $\Delta_{R E F}^{\prime}$, which is obtained in the case where the power is evenly distributed over all phases of the island and the collisional time scale is zero. Accordingly, in the context of the time dependent ECCD model described above $\Delta t_{R E F}$ is defined as

$$
\Delta_{R E F}^{\prime} \equiv \Delta_{C D}^{\prime}\left(\Delta \xi=2 \pi, \tau_{\text {coll }}=0\right) .
$$

Note that, this reference case corresponds to $\Delta_{C D}^{\prime}$ as obtained conventionally under the assumption of fast rotation and averaging the power deposition and current drive profiles over a complete rotation period. ${ }^{17,24,25}$

When the collisional time scale $\tau_{\text {coll }}$ is different from zero, the EC driven current takes time to reach a quasisteady state depending on the value of $\tau_{\text {coll. }}{ }^{26}$ As $\Delta_{C D}^{\prime}$ is proportional to $\delta J_{C D}$, it also evolves on the time scale of $\tau_{\text {coll }}$, finally reaching a quasi-steady state as shown in Fig. 2. For both curves, $\tau_{\text {coll }}=3 \tau_{\text {rot }}$. The solid curve represents the case for which the power is spread evenly over all phases of the island and shows the evolution of $\Delta_{C D}^{\prime}$ with the growth of the driven current on the collisional time scale. For $t \gg \tau_{\text {coll }}$, a steady state is reached identical to $\Delta_{R E F}^{\prime}$. Results for $\Delta_{C D}^{\prime}$ calculations with a small helical power deposition width $(0.02 \times 2 \pi)$ are depicted by the dashed curve. The helically localized power deposition on a rotating island generates a time varying phase of power deposition and current generation. That further results in oscillations of the driven current density profile and accordingly of $\Delta_{C D}^{\prime}$ at the plasma rotation frequency. For $t \gg \tau_{\text {coll }}$, a quasi-steady state is reached 


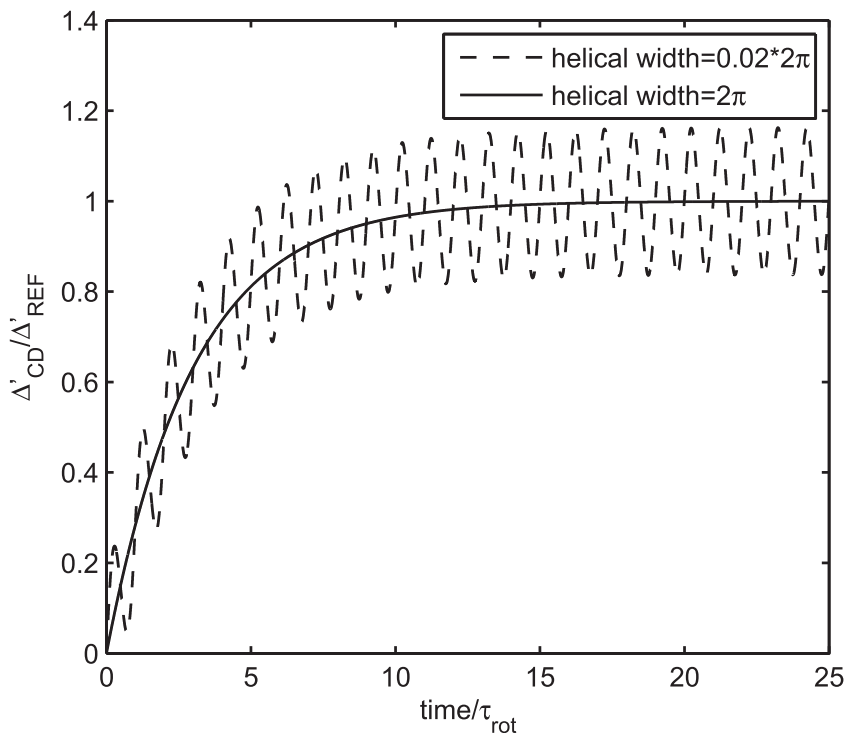

FIG. 2. $\Delta_{C D}^{\prime}$ calculations for $\tau_{\text {coll }}=3 \tau_{\text {rot }}$ as a function of time till a quasisteady state is reached. The result of the case where the power is distributed evenly over all phases of the island is given by the solid curve. The dashed curve represents $\Delta_{C D}^{\prime}$ calculations done for a power deposition helical width $2 \%$ of the whole island helical width.

characterized by periodic oscillations of $\Delta_{C D}^{\prime}$ around its reference value.

To illustrate the consequences of a different value of $\tau_{\text {coll }} / \tau_{\text {rot }}, \Delta_{C D}^{\prime}$ calculations in case of small helical width $(0.02 \times 2 \pi)$ and $w=w_{\text {dep }}$ were done for a number of $\tau_{\text {coll }}$ values. The results as obtained during two rotation periods during the quasi-steady state are shown in Fig. 3. When the rotation period is of the same order, or longer than the collisional time scale $\tau_{\text {coll }}$, as presented by the full, dashed, and dashed-dotted curves, $\Delta_{C D}^{\prime}$ oscillates with the rotation period. When $\tau_{\text {coll }} / \tau_{\text {rot }}=\infty$ (dotted curve), the oscillations of $\Delta_{C D}^{\prime}$ are reduced to zero and the quasi-steady state reached is identical to the reference case. The case of $\tau_{\text {coll }} / \tau_{\text {rot }}=0$ can

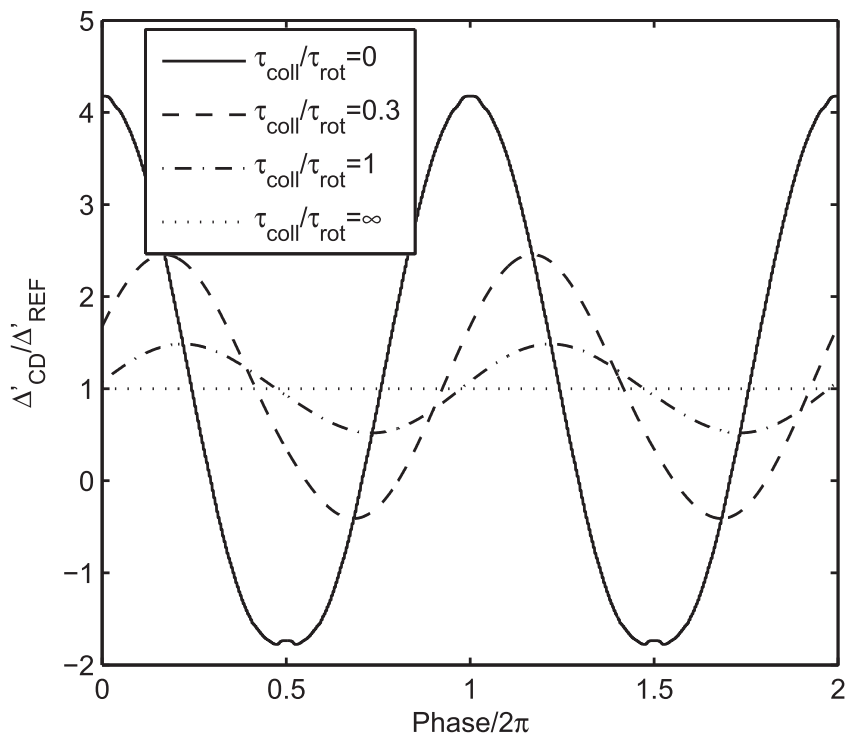

FIG. 3. Results during the quasi-steady state for $\Delta_{C D}^{\prime}$ as a function of the phase of the rotating island for $\Delta \xi=0.02 \times 2 \pi, w=w_{d e p}$, and various values of $\tau_{\text {coll }} / \tau_{\text {rot }}$ (see legend). also be seen as representing the $\Delta_{C D}^{\prime}$ efficiency as a function of island phase in case of a locked mode. Note that, in particular for small $\tau_{\text {coll }} / \tau_{\text {rot }}$, the oscillations are not sinusoidal and that the maxima and minima are not symmetric around the average value. This is a consequence of the finite width of the flux surface averaged power deposition and driven current density profiles and the particular shape of the effectiveness for NTM stabilization of the driven current as a function of the flux coordinate as presented in Fig. 18 of Giruzzi et al. ${ }^{26}$ Because of this non-sinusoidal character of the oscillations, in the following, results will be shown in terms of extrema of the oscillations.

As $\tau_{\text {coll }} / \tau_{\text {rot }}$ increases, the amplitude of the oscillations decreases as the driven current profile gets more smeared out due to the delayed generation and decay. In addition, this delay results in a phase shift of the $\Delta_{C D}^{\prime}$ oscillation relative to the island rotation as can be seen in Fig. 3. The phase shift increases with increasing $\tau_{\text {coll }}$, reaching about $80^{\circ}$ for $\tau_{\text {coll }}=\tau_{\text {rot }}$ and saturating at $90^{\circ}$ for large $\tau_{\text {coll }} / \tau_{\text {rot }}$. Note that, as Eqs. (5) and (15) are linear in case of constant island width $w$, in all cases shown above the time average over a
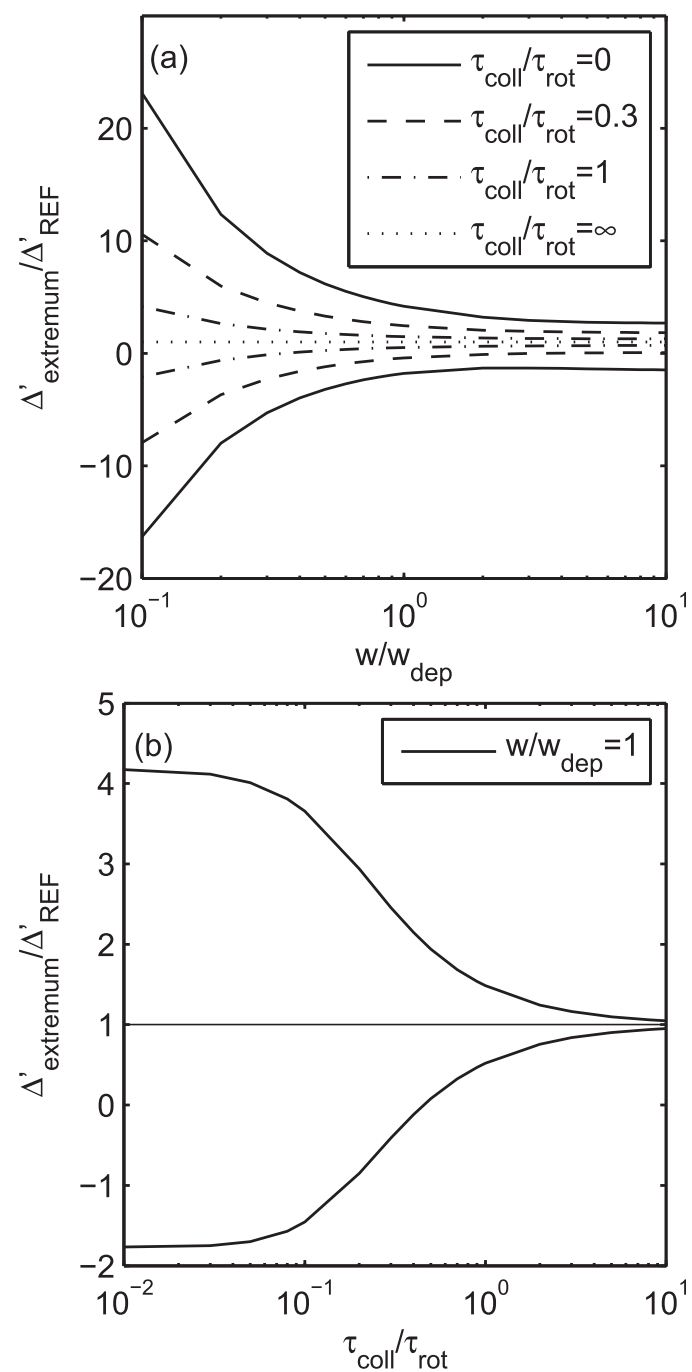

FIG. 4. (a) The dependence of extrema of $\Delta_{C D}^{\prime}$ on $w / w_{\text {dep }}$ is calculated for different $\tau_{\text {coll }} / \tau_{\text {rot }}$ values. The dotted line for which the $\tau_{\text {coll }} / \tau_{\text {rot }}=\infty$ is identical to the reference case. (b) Extrema of $\Delta_{C D}^{\prime}$ for a fixed island width $\left(w=w_{\text {dep }}\right)$ as a function of $\tau_{\text {coll }} / \tau_{\text {rot }}$. 
full rotation period of the CD term, $\left\langle\Delta_{C D}^{\prime}\right\rangle_{\tau_{\text {rot }}}$, in the long time limit of the quasi steady state is identical to the reference case, i.e.,

$$
\lim _{t \rightarrow \infty}\left\langle\Delta_{C D}^{\prime}\right\rangle_{\tau_{\text {rot }}}=\Delta_{R E F}
$$

This is indeed born out by the numerical calculations up to numerical accuracy.

The extrema of $\Delta_{C D}^{\prime}$ simulations at quasi-steady state as a function of $w / w_{\text {dep }}$ for three different $\tau_{\text {coll }} / \tau_{\text {rot }}$ are shown in Fig. 4(a). Since $\tau_{\text {coll }} / \tau_{\text {rot }}=0$ is realized in the limit of $\tau_{\text {rot }} \rightarrow \infty$ corresponding to a locked mode, the maximum of $\Delta_{C D}^{\prime}$ for $\tau_{\text {coll }} / \tau_{\text {rot }}=0$ represents the case where the power deposition is locked at the O-point, whereas its minimum refers to the power deposition locked at the X-point of the island. The amplitude of the oscillations decreases as $\tau_{\text {coll }} / \tau_{\text {rot }}$ increases. When $\tau_{\text {coll }}$ is increased from 0 to $0.3 \times \tau_{\text {rot }}$, the amplitude of the oscillations drops by about $50 \%$ for $w=0.1 \times w_{\text {dep }}$ and still by about $30 \%$ for $w=10 \times w_{\text {dep }}$. Moreover, we see that for a given value of $\tau_{\text {coll }} / \tau_{\text {rot }}$, the amplitude of the oscillations decreases as $w / w_{d e p}$ increases. Fig. 4 (b) gives the maximum and the
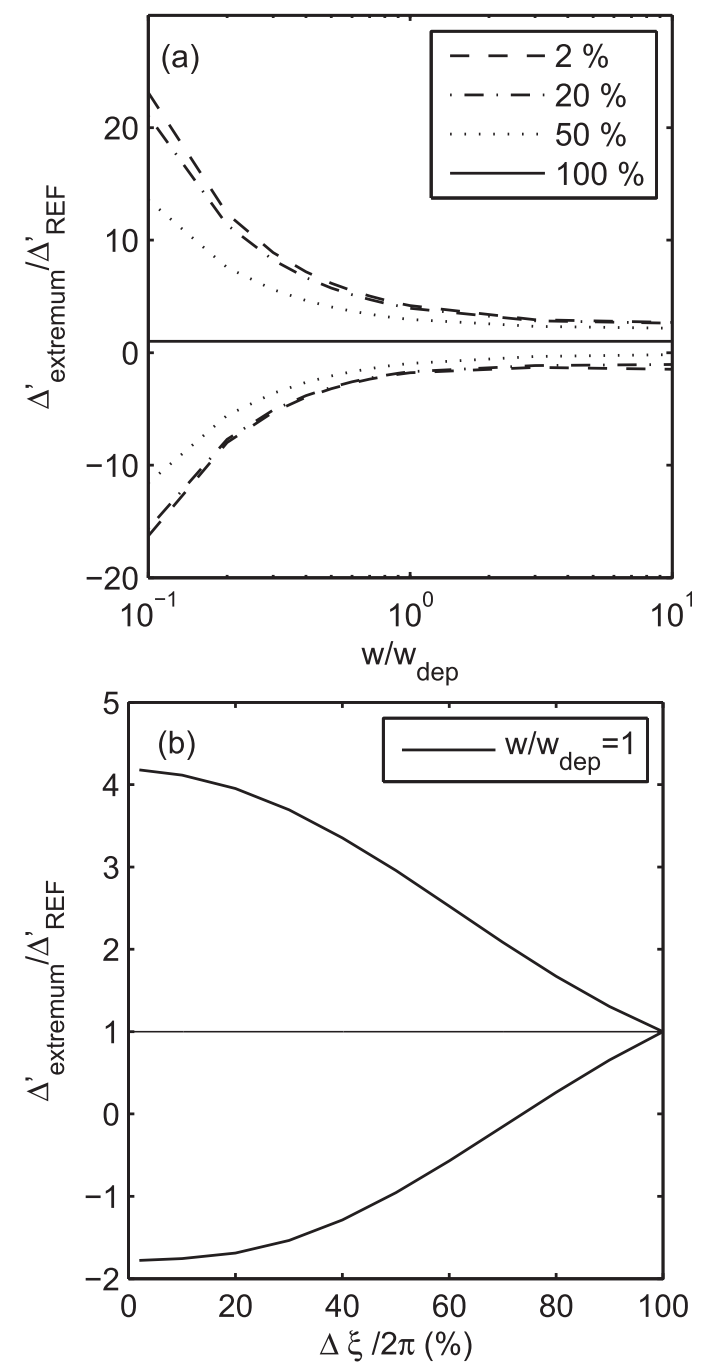

FIG. 5. In (a), the dependence of the extrema in $\Delta_{C D}^{\prime}$ on $w / w_{\text {dep }}$ is shown for different helical widths $\Delta \xi$. In each case, $\tau_{\text {coll }} / \tau_{\text {rot }}=0$. (b) Extrema of $\Delta_{C D}^{\prime}$ as a function of helical width for a fixed island width $\left(w=w_{\text {dep }}\right)$. minimum as a function of $\tau_{\text {coll }} / \tau_{\text {rot }}$ for a fixed island width $\left(w=w_{\text {dep }}\right)$. For $\tau_{\text {coll }} / \tau_{\text {rot }}<10^{-1}$, the extrema in $\Delta_{C D}^{\prime}$ are seen to saturate at a maximum value. As $\tau_{\text {coll }} / \tau_{\text {rot }}$ is increased above $10^{-1}$, the extrema quickly approach the reference value, leaving only modest oscillations for $\tau_{\text {coll }} / \tau_{\text {rot }}>1$.

Finally, $\Delta_{C D}^{\prime}$ calculations were performed for the case of $\tau_{\text {coll }} / \tau_{\text {rot }}=0$ and different helical widths of the power deposition profile $\Delta \xi$ to assess the influence of $\Delta \xi$ on the minimum and maximum of $\Delta_{C D}^{\prime}$. Fig. 5(a) shows the results in terms of the extrema of the $\Delta_{C D}^{\prime}$ oscillations as a function of $w / w_{\text {dep }}$. For any helical width smaller than the whole island helical width, the absolute value of the extrema increases as $w / w_{d e p}$ gets smaller. For $w>w_{d e p}$, the extrema in the oscillations are almost constant. The solid curve represents the reference case $\Delta \xi=2 \pi$. In Fig. 5(b), $\Delta_{\text {extremum }}^{\prime}$ is shown for a fixed island width $\left(w=w_{d e p}\right)$ as a function of helical width. The amplitude decreases as the helical width increases. In general, $\Delta \xi$ smaller than $0.2 \times 2 \pi$ results in very similar values of the extrema. For helical widths of more than $40 \%$ of $2 \pi$, there is an almost linear decrease in the amplitude, reaching zero at $\Delta \xi=2 \pi$.

\section{RESULTS OF DYNAMICAL MODELLING WITH SELF CONSISTENT ISLAND WIDTH EVOLUTION}

In this section, it is investigated how the oscillations in $\Delta_{C D}^{\prime}$ influence the growth or the stabilization of the NTMs. This is done by simulating a selected number of cases in the 2D normalized parameter space spanned by $\tau_{\text {coll }} / \tau_{\text {rot }}$ and $\tau_{N T M} / \tau_{r o t}$ as stated in the Introduction. As indicated in Fig. 6 in this parameter space, the standard regime is recovered for $\tau_{N T M} / \tau_{\text {rot }} \gg 100$ while the region with $\tau_{N T M} / \tau_{\text {rot }} \ll 1$ can be identified with the locked mode regime. In addition, as shown in Sec. III, the $\Delta_{C D}^{\prime}$ oscillations are virtually absent for $\tau_{\text {coll }} / \tau_{\text {rot }} \gg 10$. We expect effects of the oscillation in $\Delta_{C D}^{\prime}$ on the growth or the stabilization of the island in the rest of the parameter space.

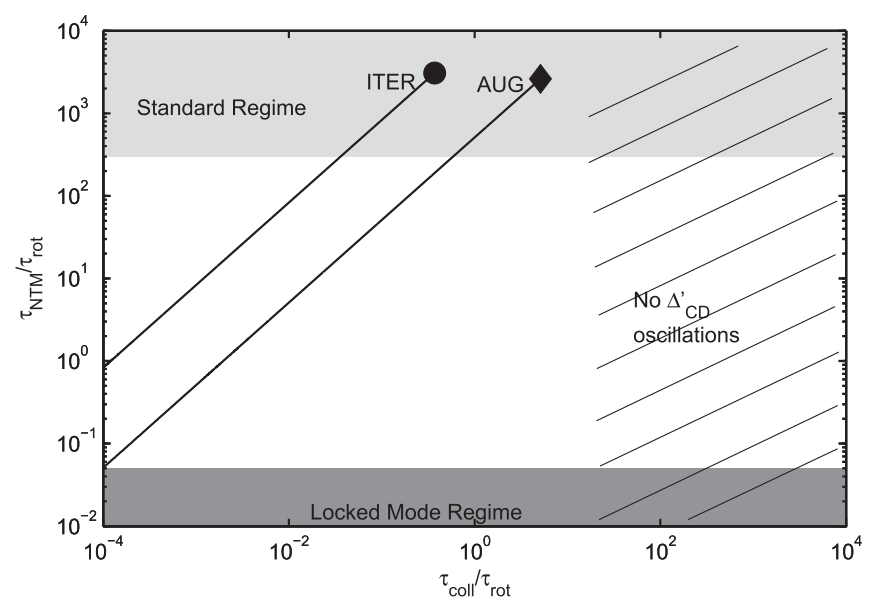

FIG. 6. The parameter space spanned by $\tau_{N T M} / \tau_{\text {rot }}$ and $\tau_{\text {coll }} / \tau_{\text {rot }}$. The fast rotation regime also called the standard regime $\left(\tau_{N T M} / \tau_{\text {rot }} \gg 100\right)$ and the locked mode regime $\left(\tau_{N T M} / \tau_{\text {rot }} \ll 1\right)$ are indicated. Beyond $\tau_{\text {coll }} / \tau_{\text {rot }} \gg 10$, the oscillations in $\Delta_{C D}^{\prime}$ are practically absent. The symbols indicate the typical position in this parameter space for an NTM as observed in a current experiment like AUG (diamond, see Table I) and as expected in ITER (circle). The lines originating from these symbols indicate the path followed in the parameter space when the rotation frequency is reduced. 
The dynamical modelling with self consistent island width evolution will be based on the GRE in its normalized form as formulated in Eq. (7). Apart from the normalized time scales, this still leaves the normalized saturated and marginal island sizes, and EC deposition width as independent parameters. For the saturated and marginal island sizes, we will be using parameters which are representative of an $m=2, n=1 \mathrm{NTM}$ in ITER: $\bar{w}_{\text {sat }}=0.2$ and $\bar{w}_{\text {marg }}=0.0125$. A case of narrow EC power deposition $\bar{w}_{d e p}=0.01$, as well as of wide deposition $\bar{w}_{d e p}=0.03$ will be considered. In all cases presented in this section, the helical EC power deposition width is given by $\Delta \xi=0.02 \times 2 \pi$.

The reference case $\left(\Delta \xi=2 \pi, \tau_{\text {coll }}=0\right)$ is used to derive a minimum required power $P_{\min }$ for NTM stabilization for both cases of narrow and wide deposition. In Figs. 7(a) and 7(b), we show the results of the simulations with an ECCD power marginally above this minimum required power for the case of $\tau_{\text {coll }} / \tau_{\text {rot }}=0$ and various values of $\tau_{N T M} / \tau_{\text {rot }}=1,10$, and 100. Compared to the reference case, the effect of rotation is an oscillation in the island width with the rotation period. The amplitude of this oscillation increases proportional to the rotation period. The oscillation results in a net increase of the stabilizing effect of the ECCD and a consequent reduction in the time required for full suppression of the mode. Note that in the case of a wide ECCD deposition, the critical island width at which the NTM growth rate attains its maximum is significantly smaller than in the case of the narrow deposition width. This can be seen in the Figs. 7(a) and 7(b) from the different island widths at which the reference cases exhibit a plateau in time. Because the oscillations in $\Delta_{C D}^{\prime}$ increase when $w / w_{\text {dep }}$ decreases, the oscillations in the island width in this plateau phase are much larger for the case with wide deposition.

In a second set of simulations, we studied the effect of introducing a finite collision time. To this end, we performed simulations with the same parameters as in case of Fig. 7(a) and $\tau_{N T M} / \tau_{\text {rot }}=10$ but now for various values of $\tau_{\text {coll }} / \tau_{\text {rot }}=0,0.01,0.1,1$, and 3 . As shown by the results given in Fig. 8, the finite collision time at first results in a further stabilizing effect of the ECCD. The stabilizing effect is seen to be largest for $\tau_{\text {coll }} / \tau_{\text {rot }} \approx 0.1$. As the collision time is increased further, the oscillations in $\Delta_{C D}^{\prime}$ and consequently in the island width are reduced.
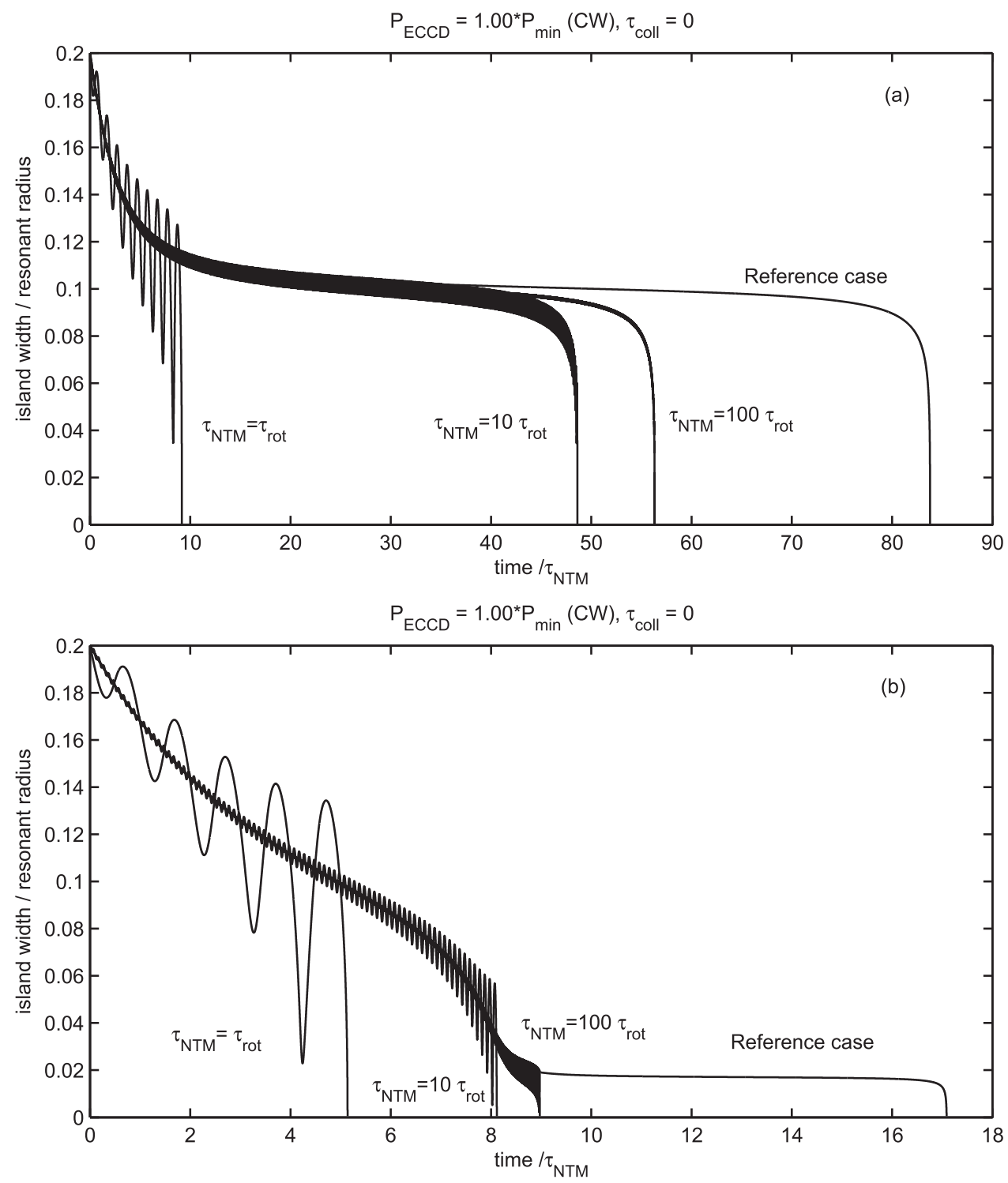

FIG. 7. The island width evolution simulated with a power marginally above $P_{\text {min }}(C W)$ for the reference case and in case of $\tau_{N T M} / \tau_{\text {rot }}=1,10$, and 100 . The other parameters are $\tau_{\text {coll }} / \tau_{\text {rot }}=0$ and $\Delta \xi=0.02 \times 2 \pi$, with either a narrow power deposition profile $\left(\bar{w}_{d e p}=0.01\right)$ (a) or a wide deposition profile $\left(\bar{w}_{d e p}\right.$ $=0.03)(\mathrm{b})$. 


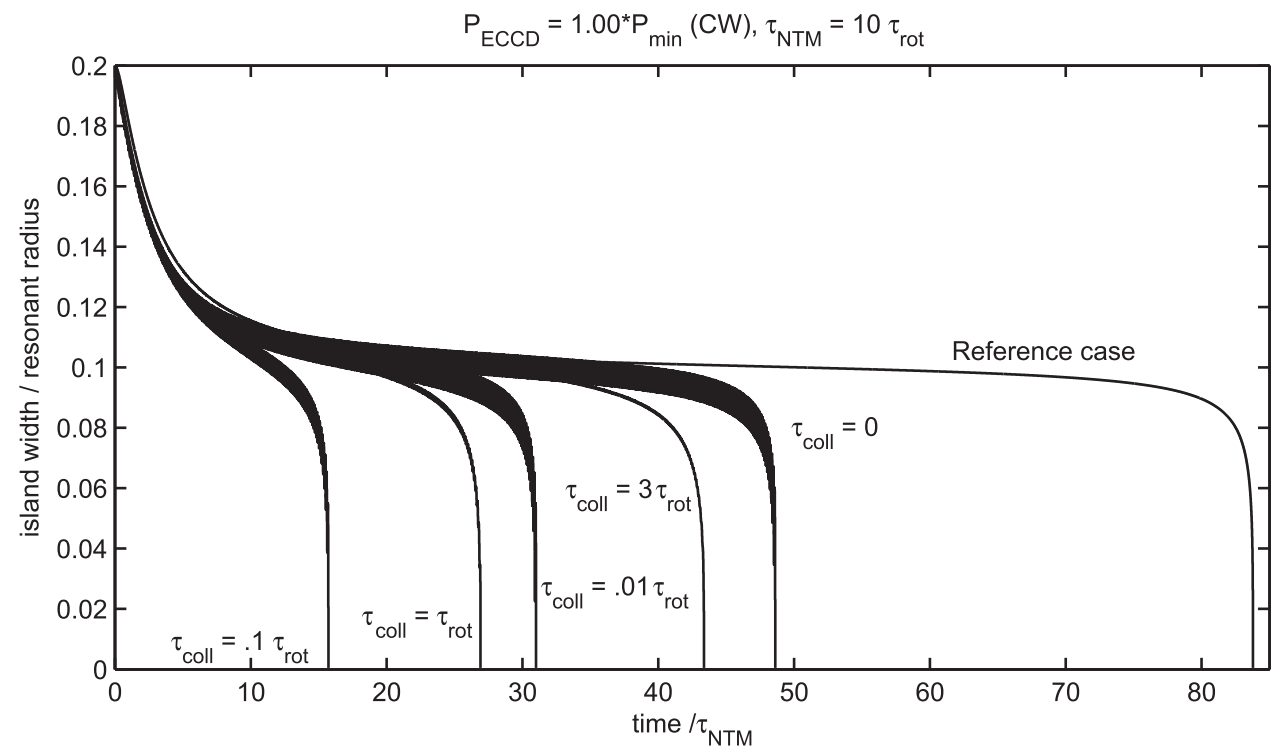

FIG. 8. The island width evolution simulated in the case of a narrow power deposition with a power marginally above the minimum $\mathrm{CW}$ requirement for a constant $\tau_{N T M} / \tau_{\text {rot }}=10$ and different $\tau_{\text {coll }} / \tau_{\text {rot }}$ values. Additionally, the evolution in the reference case is shown.

In conclusion, we see that the net result of the oscillations in $\Delta_{C D}^{\prime}$ is to increase the stabilizing effect of ECCD. As a result, also the minimum power required for full suppression is reduced below the value of $P_{\min }$ in the reference case. In case of the narrow deposition width, the minimum required power for full NTM stabilization is reduced to $95 \%$ of $P_{\text {min }}$ in case of $\tau_{N T M} / \tau_{\text {rot }}=1$ and $\tau_{\text {coll }} / \tau_{\text {rot }}=0$. For larger values of $\tau_{N T M} / \tau_{\text {rot }} \geq 10$, the reduction of the minimum required power is very small. The smaller critical island width in the case of wide ECCD deposition leads to a larger reduction in the minimum required power. In that case, the minimum required power decreases to about $90 \%$ of $P_{\min }$ for both $\tau_{N T M} / \tau_{\text {rot }}=1$, or 10 and for collision times up to $\tau_{\text {coll }} / \tau_{\text {rot }}=1.0$ or even larger in the case of $\tau_{N T M} / \tau_{\text {rot }}=1$.

We have also performed simulations for the case of modulated ECCD with $\mathcal{D}=0.5$ corresponding to a dutycycle of $50 \%$. In this case, the comparison has been made to a reference value of $\Delta_{C D}^{\prime}$ given by

$$
\Delta_{C D, R E F}^{\prime}(\mathcal{D})=\mathcal{D} \Delta_{C D}^{\prime}\left(\Delta \xi=2 \mathcal{D} \pi, \tau_{\text {rot }}=\infty, \tau_{\text {coll }}=0\right)
$$

assuming that the power is localized symmetrically around the O-point. The factor $\mathcal{D}$ originates from the fact that the power is applied only during this fraction of time. In the case of modulated power, the minimum required power $P_{\min }$ in the reference case (in terms of the required power during the power-on-time) is already lower than for the $\mathrm{CW}$ case, in particular for the wide deposition case. In this case, the $\Delta_{C D}^{\prime}$ oscillations introduced by the rotation have not been found to lead to a significant further reduction in the minimum required power.

\section{A. Application to current and future experiments}

As a typical example of present day experiments on ECCD control of NTMs, we consider the parameters of $m=3, n=2$ NTM control in ASDEX Upgrade (major radius $R_{0}=1.65 \mathrm{~m}$, minor radius $a=0.49 \mathrm{~m}$, and elongation $\kappa=1.76)$ as listed in Table $\mathrm{I}^{34}$ These parameters are characterized by a high mode rotation frequency of around $20 \mathrm{kHz}$, which is typical of present day high performance experiments with dominant heating through neutral beam injection. This puts these experiments well into the standard regime as indicated in Fig. 6. However, when the mode rotation frequency is reduced, for example, through balanced neutral beam injection or through coupling of the mode to a slowly rotating resonant magnetic perturbation, experiments could be performed in the regime studied in this paper. AUG simulations show that the effect of the oscillations in $\Delta_{C D}^{\prime}$ on the suppression of NTMs by ECCD as caused by the slower rotation is minimal up to very low rotation frequencies. Only for the case of wide ECCD deposition and when the rotation frequency is as slow as $f_{\text {rot }}=10 \mathrm{~Hz}$ (i.e., for $\tau_{N T M} / \tau_{\text {rot }} \approx 1$ ), there is a significant decrease in the minimum required power. In that case as shown in Fig. 9, full island suppression can be achieved with a power decreased to $85 \%$ of the value the reference model predicts.

In spite of the expected much lower plasma rotation frequency in ITER, the expected parameters for the ITER standard $\mathrm{H}$-mode scenario 2 still place ITER $^{35,36}$ well into the standard regime (see Fig. 6). However, it has been noticed that once an NTM is seeded in ITER, the wall induced drag will quickly slow down the mode until it locks to the error field. ${ }^{12,37-39}$ During this slow down, the discharge will move from the standard regime to the locked mode regime crossing the parameter space studied in this

TABLE I. Typical parameters for a 3/2 NTM in AUG. ${ }^{34}$

\begin{tabular}{lcc}
\hline \hline$\tau_{\text {coll }}$ & {$[\mathrm{ms}]$} & 0.257 \\
$\tau_{N T M}$ & {$[\mathrm{~s}]$} & 0.1327 \\
$\tau_{\text {rot }}$ & {$[\mathrm{ms}]$} & 1 \\
$r_{\text {res }}$ & {$[\mathrm{m}]$} & 0.24 \\
$w_{\text {dep }}$ & {$[\mathrm{m}]$} & 0.05 \\
$w_{\text {sat }}$ & {$[\mathrm{m}]$} & 0.05 \\
$w_{\text {marg }}$ & {$[\mathrm{m}]$} & 0.025 \\
$\eta_{C D}$ & {$[\mathrm{~A} / \mathrm{W}]$} & 0.006 \\
$L_{q}$ & {$[\mathrm{~m}]$} & 0.3 \\
$T_{e}$ & {$[\mathrm{keV}]$} & 2 \\
$J_{B S}$ & {$\left[\mathrm{MA} / \mathrm{m}^{2}\right]$} & 0.2 \\
$Z_{\text {eff }}$ & & 2 \\
\hline \hline
\end{tabular}




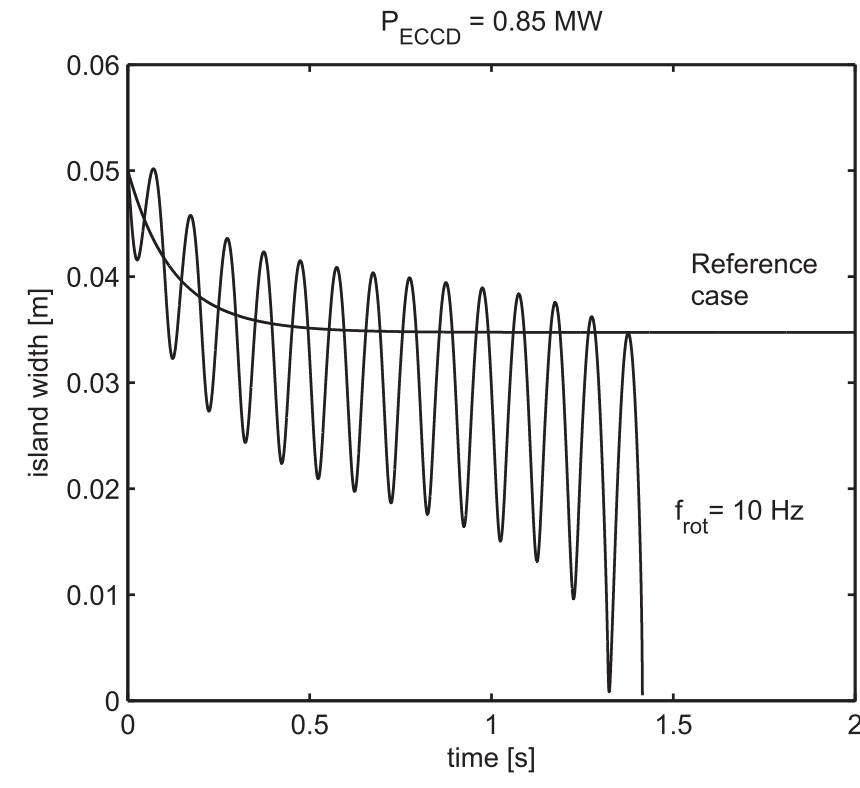

FIG. 9. Simulation results for AUG in the case of a relatively wide ECCD deposition of $w_{\text {dep }}=0.05 \mathrm{~m}$ and a low rotation frequency of $f_{\text {rot }}=10 \mathrm{~Hz}$. The simulations show that full island suppression is possible with a power of $85 \%$ of $P_{\min }(\mathrm{CW})$, which equals about $1.0 \mathrm{MW}$ for the parameters used in these simulations.

paper. In particular in the final stages just before mode locking, significant effects from the $\Delta_{C D}^{\prime}$ oscillations are to be expected. A proper analysis of this phase will require the simultaneous solution of the evolution of the mode rotation $^{38,40}$ with the evolution of the mode width as described by the dynamical model developed in this paper. This is left for future study.

\section{CONCLUSION}

In this paper, the oscillations in $\Delta_{C D}^{\prime}$ that originate from the rotation of the magnetic island through the EC power deposition region and the finite collision time relevant to the EC current drive are studied. In particular, their dependence on $\tau_{\text {coll }}, \tau_{\text {rot }}$, the island size, and the power deposition width in both radial and helical directions are investigated. Furthermore, their consequences for the island growth or stabilization are analyzed. This work shows that the net result of the oscillations in $\Delta_{C D}^{\prime}$ in combination with the consistent island size evolution is a slight increase in the stabilizing effect of ECCD averaged over a rotation period and consequently, a reduction of the minimum power required to fully suppress an island. The magnitude of this effect depends on the values of $\tau_{\text {coll }} / \tau_{\text {rot }}$ and $\tau_{N T M} / \tau_{\text {rot }}$.

The $\Delta_{C D}^{\prime}$ calculations performed to see the influence of $\tau_{\text {coll }} / \tau_{\text {rot }}$ on its time dependence show that as $\Delta_{C D}^{\prime}$ is proportional to $\delta J_{C D}$, it evolves on the time scale of $\tau_{\text {coll }}$. It takes time for $\Delta_{C D}^{\prime}$ to reach a quasi-steady state depending on the value of $\tau_{\text {coll }}$. The helically localized power deposition on a rotating magnetic island leads to oscillations at the plasma rotation frequency both in the driven current density and in $\Delta_{C D}^{\prime}$. Once the quasi-steady state is reached for $t \gg \tau_{c o l l}$, $\Delta_{C D}^{\prime}$ oscillates around a reference value, which is defined by the value of $\Delta_{C D}^{\prime}$ as obtained conventionally by averaging the power deposition over the rotation period and assuming an instantaneously driven current. Because the current drive efficiency is assumed to be linear, such that the driven current is simply proportional to the absorbed power, the time average over a full rotation period of $\Delta_{C D}^{\prime}$ during the quasisteady state is exactly equal to its reference value regardless of $\tau_{\text {coll }} / \tau_{\text {rot }}$. The amplitude of the oscillations in $\Delta_{C D}^{\prime}$ is largest for $\tau_{\text {coll }}=0$ and decreases for increasing $\tau_{\text {coll }} / \tau_{\text {rot }}$. The oscillations in $\Delta_{C D}^{\prime}$ are negligible for $\tau_{\text {coll }} / \tau_{\text {rot }} \gg 10$. For finite collision times, the oscillation in $\Delta_{C D}^{\prime}$ is shifted in phase with respect to the island rotation. For large $\tau_{\text {coll }} / \tau_{\text {rot }}$, this phase shift reaches a maximum of $90^{\circ}$. The case of $\tau_{\text {coll }} / \tau_{\text {rot }}=0$ also represents the efficiency of $\Delta_{C D}^{\prime}$ as a function of island phase for a locked mode. The oscillations in $\Delta_{C D}^{\prime}$ are affected by the helical width $\Delta \xi$ as well. The amplitude of these oscillations increases as the helical width decreases. The oscillation amplitude does not increase significantly further when the helical width is reduced below $\Delta \xi \leq 0.2 \times 2 \pi$. For a narrow helical deposition width, $\tau_{\text {coll }}=0$, and $w / w_{\text {dep }} \geq 1$, the amplitude of the oscillations in $\Delta_{C D}^{\prime}$ is almost constant at about twice the value of $\Delta_{R E F}^{\prime}$. For $w / w_{\text {dep }}<1$, the amplitude of the oscillations increases strongly with decreasing island width.

The dynamical modelling with self consistent island width evolution shows that the effect of the oscillation in $\Delta_{C D}^{\prime}$ with the rotation period is an oscillation in the island width. A decrease in the NTM rotation frequency increases the amplitude of this oscillation. The oscillation of the island width results in a small net increase of the average stabilizing effect of the ECCD and decreases the time required for full suppression of the mode. Introducing a finite collision time at first provides an extra stabilizing effect. A further increase in the collision time lowers the oscillations in $\Delta_{C D}^{\prime}$ and also in the island width. As the oscillations in $\Delta_{C D}^{\prime}$ result in an increase in the stabilization efficiency of the ECCD, the minimum power required for full suppression is reduced below the value of $P_{\min }$ in the reference case. The reduction is larger in case of wide ECCD deposition. On the other hand, in the case of modulated ECCD with a duty-cycle of $50 \%$, the net effect of the $\Delta_{C D}^{\prime}$ oscillations introduced by the rotation and ECCD modulation is insignificant and consequently does not result in a reduction of the minimum required power below the reduction that is already achieved in the reference case as a consequence of the power modulation. The application of this model to AUG experiments shows that for a sufficiently wide ECCD deposition, the minimum required power is reduced to approximately $85 \%$ of its reference value when the NTM rotation frequency is dropped to about $10 \mathrm{~Hz}$ from its natural value of about $20 \mathrm{kHz}$. To judge the effects of the oscillations in $\Delta_{C D}^{\prime}$ on the island suppression properly in the case of ITER, the dynamical modelling of ECCD with a self consistent island width evolution should be combined with a proper model of the mode rotation, which in ITER will slow down and lock on a time scale comparable to the island width evolution, once an NTM is triggered. ${ }^{38,40}$ This may also require a self consistent evaluation of the power deposition profile inside the magnetic island as obtained by ray-tracing in the presence of a magnetic island, ${ }^{41}$ and an account of possible effects from asymmetries in the shape and phase of the island. ${ }^{34,42,43}$ Future 
work will also address the conditions for and consequences of non-linear effects in the current drive efficiency at high EC power densities.

\section{ACKNOWLEDGMENTS}

This work, supported by the European Communities under the contract of Association between EURATOM/ FOM, was carried out within the framework of the European Fusion Program. The views and opinions expressed herein do not necessarily reflect those of the European Commission. The work in this paper has been performed in the framework of the NWO-RFBR Centre of Excellence on Fusion Physics and Technology (Grant 047.018.002). Discussions with F. C. Schüller are gratefully acknowledged.

${ }^{1}$ O. Sauter, R. J. La Haye, Z. Chang, D. A. Gates, Y. Kamada, H. Zohm, A. Bondeson, D. Boucher, J. D. Callen, M. S. Chu, T. A. Gianakon, O. Gruber, R. W. Harvey, C. C. Hegna, L. L. Lao, D. A. Monticello, F. Perkins, A. Pletzer, A. H. Reiman, M. Rosenbluth, E. J. Strait, T. S. Taylor, A. D. Turnbull, F. Waelbroeck, J. C. Wesley, H. R. Wilson, and R. Yoshino, "Beta limits in long-pulse tokamak discharges," Phys. Plasmas 4, 1654-1664 (1997).

${ }^{2}$ R. L. Haye, "Neoclassical tearing modes and their control," Phys. Plasmas 13, 055501 (2006).

${ }^{3}$ R. Prater, "Heating and current drive by electron cyclotron waves," Phys. Plasmas 11, 2349 (2004).

${ }^{4}$ D. Kislov, V. Alikaev, Y. Esipchuk, A. Kakurtn, A. Kislov, D. Martynov, G. Notkin, K. Razumova, A. Sushkov, and V. Volkov, "The $\mathrm{m}=2, \mathrm{n}=1$ mode suppression by ECRH on the T-10 tokamak," Nucl. Fusion 37, 339 (1997).

${ }^{5}$ E. Westerhof, A. Lazaros, E. Farshi, M. de Baar, M. de Bock, I. Classen, R. Jaspers, G. Hogeweij, H. Koslowski, A. Krämer-Flecken, Y. Liang, N. L. Cardozo, and O. Zimmermann, "Tearing mode stabilization by electron cyclotron resonance heating demonstrated in the TEXTOR tokamak and the implication for ITER," Nucl. Fusion 47, 85-90 (2007).

${ }^{6}$ I. Classen, E. Westerhof, C. Domier, A. Donné, R. Jaspers, N. Luhmann, H. Park, M. van de Pol, G. Spakman, M. Jakubowski, and TEXTOR team, "Effect of heating on the suppression of tearing modes in tokamaks," Phys. Rev. Lett. 98, 035001 (2007).

${ }^{7}$ H. Zohm, G. Gantenbein, G. Giruzzi, S. Günter, F. Leuterer, M. Maraschek, J. Meskat, A. Peeters, W. Suttrop, D. Wagner, M. Zabiégo, A. U. Team, and E. Group, "Experiments on neoclassical tearing mode stabilization by ECCD in ASDEX Upgrade," Nucl. Fusion 39, 577 (1999).

${ }^{8}$ G. Gantenbein, H. Zohm, G. Giruzzi, S. Günter, F. Leuterer, M. Maraschek, J. Meskat, and Q. Yu, "Complete suppression of neoclassical tearing modes with current drive at the electron-cyclotron-resonance frequency in ASDEX Upgrade tokamak," Phys. Rev. Lett. 85, 1242-1245 (2000).

${ }^{9}$ A. Isayama, Y. Kamada, S. Ide, K. Hamamatsu, T. Oikawa, T. Suzuki, Y. Neyatani, T. Ozeki, Y. Ikeda, K. Kajiwara, and JT-60 team, "Complete stabilization of a tearing mode in steady state high- $\beta_{p}$ H-mode discharges by the first harmonic electron cyclotron heating/current drive on JT-60U," Plasma Phys. Controlled Fusion 42, L37 (2000).

${ }^{10}$ R. J. L. Haye, S. Günter, D. A. Humphreys, J. Lohr, T. C. Luce, M. E. Maraschek, C. C. Petty, R. Prater, J. T. Scoville, and E. J. Strait, "Control of neoclassical tearing modes in DIII-D," Phys. Plasmas 9, 2051 (2002).

${ }^{11}$ C. Petty, R. L. Haye, T. Luce, D. Humphreys, A. Hyatt, J. Lohr, R. Prater, E. Strait, and M. Wade, "Complete suppression of the $m=2 / n=1$ neoclassical tearing mode using electron cyclotron current drive in DIII-D," Nucl. Fusion 44, 243-251 (2004).

${ }^{12}$ R. L. Haye, R. Prater, R. Buttery, N. Hayashi, A. Isayama, M. Maraschek, L. Urso, and H. Zohm, "Cross machine benchmarking for ITER of neoclassical tearing mode stabilization by electron cyclotron current drive," Nucl. Fusion 46, 451-461 (2006).

${ }^{13}$ M. Maraschek, G. Gantenbein, Q. Yu, H. Zohm, S. Günter, F. Leuterer, A. Manini (ECRH Group and ASDEX Upgrade Team), "Enhancement of the stabilization efficiency of a neoclassical magnetic island by modulated electron cyclotron current drive in the ASDEX Upgrade tokamak," Phys. Rev. Lett. 98, 025005 (2007).
${ }^{14}$ A. Isayama, G. Matsunaga, T. Kobayashi, S. Moriyama, N. Oyama, Y. Sakamoto, T. Suzuki, H. Urano, N. Hayashi, Y. Kamada, T. Ozeki, Y. Hirano, L. Urso, H. Zohm, M. Maraschek, J. Hobirk, K. Nagasaki, and JT-60 Team, "Neoclassical tearing mode control using electron cyclotron current drive and magnetic island evolution in JT-60U," Nucl. Fusion 49, 055006 (2009).

${ }^{15}$ P. Rutherford, "Nonlinear growth of the tearing mode," Phys. Fluids 16, 1903 (1973).

${ }^{16}$ P. Rutherford, "Basic physical processes of toroidal fusion plasmas," in Proceedings of Course and Workshop (Varenna, 1985 (Commission of the European Communities, Brussels, 1986)) Vol. 2, p. 531.

${ }^{17} \mathrm{C}$. Hegna and J. Callen, "On the stabilization of neoclassical magnetohydrodynamic tearing modes using localized current drive or heating," Phys. Plasmas 4, 2940-2946 (1997).

${ }^{18}$ H. Wilson, "Neoclassical tearing modes," Fusion Sci. Technol. 53, 152 160 (2008).

${ }^{19}$ W. Xu and J. Callen, "Report UWPR85-5," National Technical Information Service Department No. DE6008946, 1985.

${ }^{20}$ R. Carrera, R. Hazeltine, and M. Kotschenreuther, "Island bootstrap current modification of the nonlinear dynamics of the tearing mode," Phys. Fluids 29, 899-902 (1986).

${ }^{21}$ R. J. Hastie, F. Militello, and F. Porcelli, "Nonlinear saturation of tearing mode islands," Phys. Rev. Lett. 95, 065001 (2005).

${ }^{22}$ A. H. Glasser, J. M. Greene, and J. L. Johnson, "Resistive instabilities in general toroidal plasma configurations," Phys. Fluids 18, 875 (1975).

${ }^{23}$ F. Perkins, R. Harwey, M. Mahowski, and M. Rosenbluth, "Prospects for electron cyclotron current drive stabilization of neoclassical tearing modes in ITER," in Proc. 24th EPS Conference on Plasma Physics, Berchtesgaden, 9-13 June 1997 (ECA, 1997), Vol. 21A, pp. P-III. 1017.

${ }^{24} \mathrm{O}$. Sauter, "On the contribution of local current density to neoclassical tearing mode stabilization," Phys. Plasmas 11, 4808-4813 (2004).

${ }^{25}$ D. D. Lazzari and E. Westerhof, "On the merits of heating and current drive for tearing mode stabilization," Nucl. Fusion 49, 075002 (2009).

${ }^{26}$ G. Giruzzi, M. Zabiégo, T. Gianakon, X. Garbet, A. Cardinali, and S. Bernabei, "Dynamical modelling of tearing mode stabilization by rf current drive," Nucl. Fusion 39, 107 (1999).

${ }^{27}$ R. Fitzpatrick, "Helical temperature perturbations associated with tearing modes in tokamak plasmas," Phys. Plasmas 2, 825-838 (1995).

${ }^{28} \mathrm{~N}$. Bertelli, D. De Lazzari, and E. Westerhof, "Requirements on localized current drive for the suppression of neoclassical tearing modes," Nucl. Fusion 51, 103007 (2011).

${ }^{29} \mathrm{~A}$. Mikhailovskii, "Theory of magnetic islands in tokamaks with accenting neoclassical tearing modes," Contrib. Plasma Phys. 43, 125 (2003) and references herein.

${ }^{30} \mathrm{~K}$. Yoshioka and T. M. Antonsen, Jr., "Neoclassical effects on rf current drive in tokamaks," Nucl. Fusion 26, 839 (1986).

${ }^{31}$ M. O'Brien, M. Cox, and D. Start, "Fokker-Planck studies of high power electron cyclotron heating in tokamaks," Nucl. Fusion 26, 1625 (1986).

${ }^{32} \mathrm{E}$. Westerhof, "Fokker-Planck quasi-linear codes for the study of electron cyclotron resonance heating and electron cyclotron current drive," in Conf. Proc., 9th Joint Workshop on Electron Cyclotron Emission and Electron Cyclotron Heating, Borrego Springs, CA, USA (World Scientific, Singapore, 1995), p. 3.

${ }^{33}$ N. J. Fisch, "Theory of current-drive in plasmas," Rev. Mod. Phys. 59, 175-234 (1987).

${ }^{34}$ L. Urso, H. Zohm, A. Isayama, M. Maraschek, E. Poli, A. U. Team, and J. Team, "ASDEX Upgrade JT-60U comparison and ECRH power requirements for NTM stabilization in ITER," Nucl. Fusion 50, 025010 (2010).

${ }^{35}$ M. Shimada, D. Campbell, V. Mukhovatov, M. Fujiwara, N. Kirneva, K. Lackner, M. Nagami, V. Pustovitov, N. Uckan, J. Wesley, N. Asakura, A. Costley, A. Donn, E. Doyle, A. Fasoli, C. Gormezano, Y. Gribov, O. Gruber, T. Hender, W. Houlberg, S. Ide, Y. Kamada, A. Leonard, B. Lipschultz, A. Loarte, K. Miyamoto, V. Mukhovatov, T. Osborne, A. Polevoi, and A. Sips, "Chapter 1: Overview and summary," Nucl. Fusion 47, S1 (2007).

${ }^{36}$ R. Prater, D. Farina, Y. Gribov, R. Harvey, A. Ram, Y.-R. Lin-Liu, E. Poli, A. Smirnov, F. Volpe, E. Westerhof, A. Zvonkov, and ITPA Steady State Operation Topical Group, "Benchmarking of codes for electron cyclotron heating and electron cyclotron current drive under ITER conditions," Nucl. Fusion 48, 035006 (2008).

${ }^{37}$ M. Nave and J. Wesson, "Mode locking in tokamaks," Nucl. Fusion 30, 2575 (1990).

${ }^{38}$ G. Ramponi, E. Lazzaro, and S. Nowak, "On the stabilization of neoclassical tearing modes by electron cyclotron waves," Phys. Plasmas 6, 35613570 (1999). 
${ }^{39} \mathrm{Q}$. Yu and S. Günter, "Locking of neoclassical tearing modes by error fields and its stabilization by RF current," Nucl. Fusion 48, 065004 (2008).

${ }^{40}$ H. van den Brand, M. de Baar, N. L. Cardozo, and E. Westerhof, "Integrated modelling of island growth, stabilization, and mode locking: Consequences for NTM control on ITER," Plasma Phys. Controlled Fusion 54, 094003 (2012).

${ }^{41}$ H. Isliker, I. Chatziantonaki, C. Tsironis, and L. Vlahos, "Electron-cyclotron wave propagation, absorption, and current drive in the presence of neoclassical tearing modes," Plasma Phys. Controlled Fusion 54, 095005 (2012).

${ }^{42}$ E. Lazzaro and S. Nowak, "Eccd control of dynamics of asymmetric magnetic islands in a sheared flow," Plasma Phys. Controlled Fusion 51, 035005 (2009).

${ }^{43}$ D. D. Lazzari and E. Westerhof, "The role of asymmetries in the growth and suppression of neoclassical tearing modes," Plasma Phys. Controlled Fusion 53, 035020 (2011). 